\title{
ON THE CONVERGENCE OF CERTAIN PARTIAL SUMS OF A TAYLOR SERIES WITH GAPS
}

\author{
GEORGE PIRANIAN
}

We consider the function $f(z)$ determined by the power series

$$
f(z)=\sum_{1}^{\infty} c_{n} z^{\lambda_{n}}
$$

and its direct analytic continuation. For simplicity, it is supposed that $\lim \sup \left|c_{n}\right|^{1 / \lambda_{n}}=1$.

We write

$$
\begin{aligned}
S_{n}(z) & =\sum_{1}^{n} c_{p} z^{\lambda_{p}}, & \\
M(r) & =\max _{|z|=r}|f(z)| & (0<r<1), \\
M(r) & =1 & (r \leqq 0), \\
\theta_{n} & =\lambda_{n+1} / \lambda_{n}-1 . &
\end{aligned}
$$

Ostrowski has proved ${ }^{1}$ that if $\left\{\theta_{n_{i}}\right\}$ is a sequence extracted from the sequence $\left\{\theta_{n}\right\}$ such that $\lim \inf \theta_{n_{i}}>0$, then every regular point of $f(z)$ on the circle $|z|=1$ is the center of a circle in which the sequence $\left\{S_{n_{i}}(z)\right\}$ converges uniformly to $f(z)$. Restricting ourselves to the question of convergence at the regular points themselves, we shall prove the following theorem:

If

$$
\limsup _{i \rightarrow \infty} \frac{\log \left(M\left(1-\theta_{n_{i}}^{2}\right) / \theta_{n_{i}}\right)}{\lambda_{n_{i}} \theta_{n_{i}}^{2}}<\infty,
$$

then $\lim S_{n_{i}}(z)=f(z)$ at all regular points of (1) on the circle $|z|=1$.

For the proof, we shall assume that $\lim \theta_{n_{i}}=0$; afterwards, we shall remove this restriction, with the aid of Ostrowski's theorem.

Let $z_{1}$ be a regular point for (1) on the circle $|z|=1$, and let $z_{0}$ be a point on the segment joining $z_{1}$ to the origin. We write $\left|z_{1}-z_{0}\right|=a$, and for every positive integer $i$ we define the three circles

Presented to the Society, April 24, 1943; received by the editors January 8, 1943.

1 A. Ostrowski, Über eine Eigenschaft gewisser Potenzreihen mit unendlich vielen verschwindenden Koefficienten. Preuss. Akad. Wiss. Sitzungsber. vol. 34 (1921) pp. 557565. Essentially the same proof is to be found in P. Montel's Leçons sur les families normales de fonctions analytiques et leurs applications, pp. 204-207. 


$$
\begin{array}{cc}
\Gamma_{i}^{\prime}:\left|z-z_{0}\right|=\rho_{i}^{\prime} & \left(0<\rho_{i}^{\prime}<a\right), \\
\Gamma(a):\left|z-z_{0}\right|=a, \quad \Gamma_{i}^{\prime \prime}:\left|z-z_{0}\right|=\rho_{i}^{\prime \prime} & \left(\rho_{i}^{\prime \prime}>a\right),
\end{array}
$$

where $\rho_{i}^{\prime \prime}$ shall be chosen so that the function $f(z)$ is holomorphic in the closed region bounded by $\Gamma_{i}^{\prime \prime}$. By $M_{i}^{\prime}, M_{i}(a)$, and $M_{i}^{\prime \prime}$ we denote the maximum value of $\left|f(z)-S_{n_{i}}(z)\right|$ on $\Gamma_{i}^{\prime}, \Gamma(a)$, and $\Gamma_{i}^{\prime \prime}$, respectively. We write

$$
R_{i}^{\prime}=1+\rho_{i}^{\prime}-a, \quad R_{i}^{\prime \prime}=1+\rho_{i}^{\prime \prime}-a,
$$

and we choose $r_{i}$ so that $R_{i}^{\prime}<r_{i}<1$.

By Cauchy's formula we have, on the circle $|z|=R_{i}^{\prime}$,

$$
\left|f(z)-S_{n_{i}}(z)\right| \leqq \sum_{p=\lambda_{n_{i}+1}}^{\infty} \frac{M\left(r_{i}\right)}{r_{i}^{p}} R_{i}^{\prime p}=\frac{M\left(r_{i}\right) r_{i}}{r_{i}-R_{i}^{\prime}}\left(\frac{R_{i}^{\prime}}{r_{i}}\right)^{\lambda_{n_{i}}\left(1+\theta_{n_{i}}\right)},
$$

and by the principle of the maximum

$$
\log M_{i}^{\prime} \leqq \log \frac{M\left(r_{i}\right) r_{i}}{r_{i}-R_{i}^{\prime}}+\lambda_{n_{i}}\left(1+\theta_{n_{i}}\right) \log \frac{R_{i}^{\prime}}{r_{i}} .
$$

On the circle $|z|=R_{i}^{\prime \prime}$, and therefore also on $\Gamma_{i}^{\prime \prime}$, we have

$$
\left|S_{n_{i}}(z)\right| \leqq M\left(r_{i}\right) \sum_{0}^{\lambda_{n i}}\left(\frac{R_{i}^{\prime \prime}}{r_{i}}\right)^{p}<\frac{M\left(r_{i}\right) R_{i}^{\prime \prime}}{R_{i}^{\prime \prime}-r_{i}}\left(\frac{R_{i}^{\prime \prime}}{r_{i}}\right)^{\lambda_{n i}} .
$$

Since $f(z)$ is holomorphic in the closed region bounded by $\Gamma_{i}^{\prime \prime}$, and since at least one of the expressions $R_{i}^{\prime \prime} /\left(R_{i}^{\prime \prime}-r_{i}\right),\left(R_{i}^{\prime \prime} / r_{i}\right)^{\lambda_{n_{i}}}$ tends to $\infty$ as $i \rightarrow \infty$, we may write, for any positive $\eta$ and for $i$ sufficiently large,

$$
\log M_{i}^{\prime \prime}<\log \frac{M\left(r_{i}\right)(1+\eta) R_{i}^{\prime \prime}}{R_{i}^{\prime \prime}-r_{i}}+\lambda_{n_{i}} \log \frac{R_{i}^{\prime \prime}}{r_{i}} .
$$

Applying Hadamard's three-circle theorem to the function $S_{n_{i}}(z)$ $-f(z)$ on the circles $\Gamma_{i}^{\prime}, \Gamma(a), \Gamma_{i}^{\prime \prime}$, we have now

$$
\begin{aligned}
\log \frac{\rho_{i}^{\prime \prime}}{\rho_{i}^{\prime}} \log M_{i}(a) \leqq & \log \left(\frac{\rho_{i}^{\prime \prime}}{a}\right) \log M_{i}^{\prime}+\log \left(\frac{a}{\rho_{i}^{\prime}}\right) \log M_{i}^{\prime \prime} \\
< & \log \left(\frac{\rho_{i}^{\prime \prime}}{a}\right)\left\{\log \frac{M\left(r_{i}\right) r_{i}}{r_{i}-R_{i}^{\prime}}+\lambda_{n_{i}}\left(1+\theta_{n_{i}}\right) \log \frac{R_{i}^{\prime}}{r_{i}}\right\} \\
+ & \log \left(\frac{a}{\rho_{i}^{\prime}}\right)\left\{\log \frac{M\left(r_{i}\right)(1+\eta) R_{i}^{\prime \prime}}{R_{i}^{\prime \prime}-r_{i}}+\lambda_{n_{i}} \log \frac{R_{i}^{\prime \prime}}{r_{i}}\right\}
\end{aligned}
$$

In the proof of his theorem Ostrowski now takes the point $z_{0}$ near 
to the point $z_{1}$ and chooses for $\Gamma_{i}^{\prime}, \Gamma(a)$, and $\Gamma_{i}^{\prime \prime}$ three fixed circles with radii sufficiently near to $\left|z_{1}-z_{0}\right|$ (with $\Gamma(a)$ including the point $z_{1}$ instead of passing through it). In our case it is necessary to take $z_{0}$ near to the origin and to let $\rho_{i}^{\prime}$ and $\rho_{i}^{\prime \prime}$ tend to $a$ as $i$ becomes large. We choose $\rho_{i}^{\prime}=\left(1-b_{i}\right) a, \rho_{i}^{\prime \prime}=\left(1+b_{i}\right) a, r_{i}=1-k_{i}$, where $0<b_{i}<1$ and $0<k_{i}<a b_{i}$; substituting these values in (3), expanding terms such as $\log \left(1+a b_{i}\right)$ in power series, and dividing both sides of the inequality by $b_{i}$, we get

$$
\begin{aligned}
& 2\left(1+b_{i}^{2} / 3+\cdots\right) \log M_{i}(a)<\left(1-b_{i} / 2+b_{i}^{2} / 3-\cdots\right) \\
& \text {. }\left\{\log \frac{M\left(1-k_{i}\right)\left(1-k_{i}\right)}{\left(1-k_{i} / a b_{i}\right) a b_{i}}+\lambda_{n_{i}}\left(1+\theta_{n_{i}}\right) \log \frac{1-a b_{i}}{1-k_{i}}\right\} \\
& +\left(1+b_{i} / 2+b_{i}^{2} / 3+\cdots\right) \\
& \left\{\log \frac{M\left(1-k_{i}\right)\left(1-k_{i}\right)(1+\eta)}{\left(1+k_{i} / a b_{i}\right) a b_{i}}+\lambda_{n_{i}} \log \frac{1+a b_{i}}{1-k_{i}}\right\} \\
& <2 \eta+3 k_{i}+3 k_{i} / a b_{i}-3 \log a \\
& +2\left(1+b_{i}^{2} / 3+\cdots\right) \log \frac{M\left(1-k_{i}\right)}{b_{i}} \\
& +\lambda_{n_{i}}\left\{\theta_{n_{i}}\left[1-b_{i} / 2+b_{i}^{2} / 3-\cdots\right]\right. \\
& \cdot\left[-a b_{i}-a^{2} b_{i}^{2} / 2-\cdots+k_{i}+k_{i}^{2} / 2+\cdots\right] \\
& +\left[1-b_{i} / 2+b_{i}^{2} / 3-\cdots\right]\left[-a b_{i}-a^{2} b_{i}^{2} / 2-\cdots\right] \\
& +\left[1+b_{i} / 2+b_{i}^{2} / 3+\cdots\right]\left[a b_{i}-a^{2} b_{i}^{2} / 2+\cdots\right] \\
& \left.+2\left[1+b_{i}^{2} / 3+\cdots\right]\left[k_{i}+k_{i}^{2} / 2+\cdots\right]\right\} \\
& <2 \eta+3 k_{i}+3 k_{i} / a b_{i}-3 \log a+3 \log \frac{M\left(1-k_{i}\right)}{b_{i}} \\
& -\left(\lambda_{n_{i}} / 2\right)\left\{a b_{i}\left[\theta_{n_{i}}-b_{i}(1-a)\right]-3 k_{i}\right\}
\end{aligned}
$$

provided $\theta_{n_{i}}$ and $b_{i}$ are sufficiently small and the sum of the terms in the braces of the last term is positive.

Now suppose that

$$
\limsup _{i \rightarrow \infty} \frac{\log \left(M\left(1-\theta_{n_{i}}^{2}\right) / \theta_{n_{i}}\right)}{\lambda_{n_{i}} \theta_{n_{i}}^{2}}<H \quad(1<H<\infty) .
$$

Choose $b_{i}=b \theta_{n_{i}}, k_{i}=b_{i} \theta_{n_{i}} / b=\theta_{n_{i}}^{2}$, where $b>14 H$. The last member of (4) becomes

$$
-\left(\lambda_{n} \theta_{n_{i}}^{2} b / 2\right)\{a[1-b(1-a)]-3 / b\}
$$


If $a$ is chosen sufficiently near to $1, b\{a[1-b(1-a)]-3 / b\}>10 H$, and for sufficiently large values of $i(4)$ becomes

$$
(2+\epsilon) \log M_{i}(a)<K(a, b)+3 \log M\left(1-\theta_{n_{i}}^{2}\right) / \theta_{n_{i}}-5 \lambda_{n_{i}} \theta_{n_{i}}^{2} H,
$$

where $K(a, b)$ depends on $a$ and $b$ only. But $\lim _{i \rightarrow \infty} \theta_{n_{i}}=0$ implies, together with the validity of (2), that $\lim _{i \rightarrow \infty} \lambda_{n_{i}} \theta_{n_{i}}^{2}=\infty$. For sufficiently large $i$ we have

$$
(2+\epsilon) \log M_{i}(a)<-\lambda_{n_{i}} \theta_{n_{i}}^{2} H
$$

that is,

$$
\lim _{i \rightarrow \infty} \log M_{i}(a)=-\infty
$$

and, in particular,

$$
\lim _{i \rightarrow \infty} S_{n_{i}}\left(z_{1}\right)=f\left(z_{1}\right) .
$$

Now let $\left\{\theta_{n_{i}}\right\}$ be any sequence of values $\theta_{n_{i}}$ for which (2) is satisfied, and let $z_{1}$ be a regular point for (1). From every subsequence of $\left\{\theta_{n_{i}}\right\}$ we can extract a further subsequence $\left\{\theta_{m_{j}}\right\}$ such that either $\lim \theta_{m_{j}}=0$ or $\lim \inf \theta_{m_{j}}>0$. In the first case, $\lim S_{m_{j}}\left(z_{1}\right)=f\left(z_{1}\right)$ by what we have just proved; in the second case, by Ostrowski's theorem. From every subsequence of $\left\{n_{i}\right\}$ we can therefore extract a further subsequence $\left\{m_{j}\right\}$ such that $\lim S_{m_{j}}\left(z_{1}\right)=f\left(z_{1}\right)$. It follows that the sequence $\left\{S_{n_{i}}\left(z_{1}\right)\right\}$ itself tends to $f\left(z_{1}\right)$, and our theorem is proved.

Condition (2) may be replaced by one that is somewhat less general, but can be expressed more immediately in terms of the behavior of $M(r)$ :

If $\lim \inf \theta_{n_{i}}=\theta$, we define, for $h>\theta$,

$$
\lambda(h)=\underset{\theta_{n_{i}} \leq h}{\text { g.l.b. }} \lambda_{n_{i}}
$$

for $0 \leqq h \leqq \theta$, we write $\lambda(h)=\infty$. It follows from our theorem that $\lim S_{n_{i}}\left(z_{1}\right)=f\left(z_{1}\right)$ whenever $z_{1}$ is a regular point for (1) on the circle $|z|=1$, provided

$$
\limsup _{h \rightarrow 0+} \frac{\log \left(M\left(1-h^{2}\right) / h\right)}{h^{2} \lambda(h)}<\infty .
$$

If we write $r=1-h^{2}$, (5) becomes

$$
\limsup _{r \rightarrow 1-0} \frac{\log \left[M(r) /(1-r)^{1 / 2}\right]}{(1-r) \lambda(1-r)^{1 / 2}}<\infty .
$$


The author is indebted to Professor S. Mandelbrojt, who suggested the existence of the present theorem and gave valuable assistance in its development.

The Rice Institute

\section{ON ABEL AND LEBESGUE SUMMABILITY}

\section{OTTO SZÁSZ}

1. Introduction. A series $\sum_{1}^{\infty} a_{n}$ is called Abel summable to the value $s$ if the power series $\sum a_{n} r^{n}$ converges for $0<r<1$, and if $\sum a_{n} r^{n} \rightarrow s$ as $r \uparrow 1$; it is called Lebesgue summable if the sine series

$$
\sum_{1}^{\infty} a_{n} \frac{\sin n t}{n}=F(t)
$$

converges in some interval $0<t<\tau$, and if

$$
t^{-1} F(t) \rightarrow s \text { as } t \downarrow 0 .
$$

We write in the first case $A \sum a_{n}=s$, and in the latter case $L \sum a_{n}=s$ (summability $A$ or $L$ respectively). It is known that convergence does not imply $L$-summability and conversely $L$-summability does not imply convergence of $\sum a_{n}$. Tauberian type problems which arise out of this situation have been discussed. ${ }^{1}$ It is also known that either convergence or $L$-summability imply $A$-summability. As to the converse (restricting ourselves to real $a_{n}$ ) we have proved the following theorems:

THEOREM 1. [8, pp. 582-583]. If

$$
\sum_{n}^{2 n}\left(\left|a_{\nu}\right|-a_{\nu}\right)=O(1) \quad \text { as } n \rightarrow \infty,
$$

and if

$$
\sum_{1}^{\infty} a_{n} r^{n}=O(1) \quad \text { as } \quad r \uparrow 1,
$$

Presented to the Society, December 27, 1942; received by the editors December $16,1942$.

${ }^{1}$ See [8], where further references are given; numbers in brackets refer to the bibliography at the end of this paper. 